\title{
Demystifying the memory effect: A geometrical approach to understanding speckle correlations ${ }^{\star}$
}

\author{
Aaron C. Prunty ${ }^{\mathrm{a}}$ and Roel K. Snieder \\ Center for Wave Phenomena, Department of Geophysics, Colorado School of Mines, \\ Golden, CO, USA
}

Received 1 September 2016 / Received in final form 5 November 2016 Published online 25 May 2017

\begin{abstract}
The memory effect has seen a surge of research into its fundamental properties and applications since its discovery by Feng et al. [Phys. Rev. Lett. 61, 834 (1988)]. While the wave trajectories for which the memory effect holds are hidden implicitly in the diffusion probability function [Phys. Rev. B 40, 737 (1989)], the physical intuition of why these trajectories satisfy the memory effect has often been masked by the derivation of the memory correlation function itself. In this paper, we explicitly derive the specific trajectories through a random medium for which the memory effect holds. Our approach shows that the memory effect follows from a simple conservation argument, which imposes geometrical constraints on the random trajectories that contribute to the memory effect. We illustrate the time-domain effects of these geometrical constraints with numerical simulations of pulse transmission through a random medium. The results of our derivation and numerical simulations are consistent with established theory and experimentation.
\end{abstract}

\section{Introduction}

Coherent wave transmission through a highly random medium, or reflection off a rough surface, results in an interference pattern known as speckle [3]. The memory effect is a phenomenon of wave propagation in which variations in the illumination, or incident wave, are preserved in the reflected or transmitted wave produced from the random medium, provided the variations are not too large. Thus, what was once thought to be a hopelessly complicated relation between the incident wave and the scattering medium, the complex and seemingly random speckle pattern has, in fact, a predictable behavior owing to the memory effect. The degree to which the changes in illumination are preserved in the reflected or transmitted wave is measured by means of a correlation. The correlation is taken between a designated reference speckle pattern and another speckle pattern produced by perturbing the incident wave.

\footnotetext{
* Supplementary material in the form of 3 gif files available from the Journal web page at: https://doi.org/10.1140/epjst/e2016-60254-0

a e-mail: prunty@mines.edu
} 
For example, such a perturbation might be the change in angle of incidence of the incoming wave.

The memory effect was originally derived by applying a diagrammatic perturbation expansion to the intensity-intensity correlation function for optical wave transmission through random media [1]. What was to be termed the "memory effect" was the leading order correlation term in this perturbation expansion (this term is also commonly referred to as the short-range angular correlation, memory correlation, or memory correlation function). The distinctive property of the memory correlation function is that it is present only if the change in angle of the outgoing wave matches the change in angle of the incident wave. This property causes the random speckle pattern to track the motion of the incident wave without decorrelating. For an incident wave with wave vector $\mathbf{k}$ that transmits through a random medium of thickness $D$, the diagrammatic calculation of Feng et al. [1] determined that the memory correlation function should asymptotically fall off for changes in angle of incidence greater than $1 / k D$, after which the speckle pattern changes. The predictions of this derivation were first experimentally verified by Freund et al. [4], who also extended the theory to include memory correlations for reflections off rough surfaces. Berkovits et al. [2] showed the physical basis of the memory effect to be the diffusive probability of a photon that enters the scattering medium at one point and exits the scattering medium at another. The diffusion probability function is maximized when the photon enters and exits the scattering medium from points near the mean scatterer position along the line of sight. This result was derived by considering the interference of all possible photon trajectories through the random medium. An important conclusion of this derivation was that the memory effect is independent of the phases acquired by the waves through the multiple scattering process. Much research has since been done on the fundamental properties and applications of the memory effect, including its generalization to polarized waves, incorporation of the effects of internal reflections and absorption within the scattering medium, and noninvasive imaging experiments [5-18].

In this paper, we offer a simple derivation which shows explicitly the trajectories through the random medium for which the memory effect holds. We derive the memory effect for the case of a point source using geometrical arguments only, without the need to invoke diagrammatic methods or special functions. The emphasis of our derivation is on the phases of the scattered waves, showing that the necessary conditions for the memory effect to hold can be found by considering path differences and a conservation argument. Additionally, while previous treatments of the memory effect have been given in the frequency domain assuming a monochromatic, continuous-wave source, we demonstrate the memory effect in the time domain using a bandlimited pulse source by performing two-dimensional numerical simulations. Although our source is bandlimited, we continue to refer to the simulated interference pattern as "speckle" for consistency with the literature.

\section{Theory}

A speckle pattern is produced by the superposition of many different scattered waves that interfere with effectively random phases. Thus, because the memory effect is a speckle phenomenon (an interference phenomenon), it can manifest in any solution to a linear wave equation. As we will show in this section, the backbone of the memory effect is simply the superposition principle, together with a few geometrical arguments.

The key principle behind the memory effect is that a particular speckle realization is the result of waves that interefere with particular phase differences. That is, the random but unique phase differences between the different scattered waves are what 


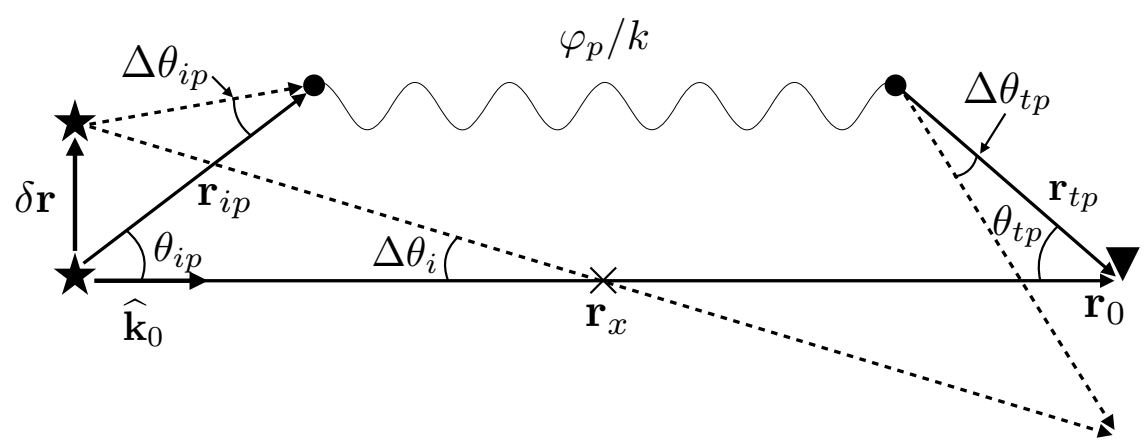

Fig. 1. Definition of the problem geometry for a point source. The solid lines indicate the original incident and transmitted waves. The dashed lines indicate the perturbed incident and transmitted waves. Arbitrary multiple scattering is represented by the wiggly line.

produce a particular speckle pattern. Intuitively, to observe the same speckle pattern after we change the angle of incidence of the incoming wave, our first condition to impose is that the phase differences amongst the different scattered waves be preserved, or held constant. This conservation argument imposes the geometrical constraints on the scattered waves that contribute to the memory effect, as we illustrate below.

Suppose we have a point source (denoted by a star in Fig. 1) that emits a pulse which transmits through a random medium. On the other side of the random medium, we observe the resultant speckle pattern at a point $\mathbf{r}_{0}$ (the point of observation denoted by an inverted triangle in Fig. 1). While a phase difference can be found between any two aribtrary waves at $\mathbf{r}_{0}$, possibly the simplest choice is to let the phase of the direct wave (i.e., the nonscattered wave that traverses the line of sight connecting the point source to the speckle spot) be the reference against which all other phases are compared. Using this choice of reference, we consider the wave function at the point $\mathbf{r}_{0}$ and for a fixed wave number $k$. The wave function is then given by a summation over all possible scattering trajectories $p$ through the random medium consisting of a finite number of isotropic point scatterers [19]:

$$
\psi\left(\mathbf{r}_{0}\right)=\sum_{p} A_{p} e^{i k L_{p}}=\sum_{p} A_{p} e^{i k\left(L_{0}+\Delta L_{p}\right)} .
$$

Here, $A_{p}$ is the complex amplitude and $L_{p}$ is the path length attributed to path $p$. Denoting the length of the line of sight trajectory by $L_{0}$, the path difference $\Delta L_{p}:=L_{p}-L_{0}$ is used to determine the phase difference between any scattered wave and the direct wave.

Let us denote by $\Delta L_{p}$ the path difference before the incident wave is perturbed, and by $\widetilde{\Delta L}_{p}$ the path difference after the incident wave is perturbed. Then, according to our conservation argument, we must impose the condition $\widetilde{\Delta L}_{p}=\Delta L_{p}$ to obtain the memory effect. It remains to determine $\Delta L_{p}$ and $\widetilde{\Delta L}_{p}$.

Figure 1 illustrates the geometry of our problem. For the direct wave, its path is characterized by the unit wave vector $\widehat{\mathbf{k}}_{0}$ along the line of sight path vector $\mathbf{r}_{0}$ (here, we have tacitly assumed the origin of our frame of reference to be the location of the point source). For any scattered wave, however, the path length is more difficult to determine. A major simplification arises when we consider the fact that a perturbation in the path length of any given trajectory through the random medium depends only on the variations in length from the source to the first scatterer and from the last scatterer to the point of observation; that is, perturbing the incident wave does 
not change the multiple scattering trajectories occurring between the first and last scattering events along a given path. This observation allows us to relegate the total phase contribution of these multiple scattering trajectories to a constant $\varphi_{p}$ for each path. Thus, to determine the path length of a scattering trajectory $p$, we need the path vector from the point source to the first scatterer, denoted by $\mathbf{r}_{i p}$, and the path vector from the last scatterer to the point of observation, denoted by $\mathbf{r}_{t p}$. By using the inner product $\langle\cdot, \cdot\rangle$, we may determine the incidence and transmission angles $\theta_{i p}$ and $\theta_{t p}$, respectively, formed between any trajectory and the line of sight (see Fig. 1).

With these considerations, the path difference before perturbing the incident wave $\Delta L_{p}$ is given by

$$
\Delta L_{p}=\left\langle\widehat{\mathbf{k}}_{0}, \mathbf{r}_{i p}\right\rangle \sec \theta_{i p}+\left\langle\widehat{\mathbf{k}}_{0}, \mathbf{r}_{t p}\right\rangle \sec \theta_{t p}+\varphi_{p} / k-\left\langle\widehat{\mathbf{k}}_{0}, \mathbf{r}_{0}\right\rangle
$$

More care needs to be taken to determine the path difference $\widetilde{\Delta L}_{p}$ after perturbing the incident wave. For a point source, we can change the angle of incidence of the incoming wave by simply perturbing the location of the source in a direction orthogonal to the line of sight (we could, in fact, perturb the source position in any arbitrary direction, but only the component orthogonal to the line of sight affects the path difference). Therefore, we let $\delta \mathbf{r}$ denote a perturbation in the source position such that $\left\langle\widehat{\mathbf{k}}_{0}, \delta \mathbf{r}\right\rangle=0$. For a scattered wave, such a perturbation in the source position results in a change in angle of incidence $\Delta \theta_{i p}$ to the first scatterer. However, as stated above, the multiple scattering trajectories between the first and last scattering events do not change as a result of $\delta \mathbf{r}$. Thus, any variations in path length caused by a change in angle of incidence must be compensated for by a change in angle of transmission $\Delta \theta_{t p}$ from the last scatterer to a new point of interference. Keeping in mind that we need to preserve the phase difference between the scattered and direct waves after perturbing the source position, we must also require the direct wave to rotate through some angle $\Delta \theta_{i}$; however, it is not yet clear about which point the direct wave must rotate. We denote this unknown rotation point by $\mathbf{r}_{x}$ (again, see Fig. 1).

Thus, by anology with equation (2), the path difference after perturbing the incident wave $\widetilde{\Delta L}_{p}$ is given by

$$
\widetilde{\Delta L}{ }_{p}=\left\langle\widehat{\mathbf{k}}_{0}, \mathbf{r}_{i p}\right\rangle \sec \left(\theta_{i p}+\Delta \theta_{i p}\right)+\left\langle\widehat{\mathbf{k}}_{0}, \mathbf{r}_{t p}\right\rangle \sec \left(\theta_{t p}+\Delta \theta_{t p}\right)+\varphi_{p} / k-\left\langle\widehat{\mathbf{k}}_{0}, \mathbf{r}_{0}\right\rangle \sec \Delta \theta_{i} .
$$

Notice that when we set $\widetilde{\Delta L}_{p}=\Delta L_{p}$, the constant $\varphi_{p} / k$ cancels, in perfect agreement with the conclusion of Berkovits et al. [2], reaffirming that the memory effect is independent of the phases acquired through the multiple scattering process. Another important characeteristic is the general form of the path difference in equations (2) and (3). In particular, the path difference depends upon $\sec \theta$, which grows very large as $\theta$ deviates from $0^{\circ}$, causing the complex exponential in equation (1) to rapidly oscillate. Thus, a path summation (integration) amounts to integrating over angles $\theta$, and its dominant contribution comes from incidence and transmission trajectories nearly parallel to the line of sight [20]. We therefore assume the angles in equations (2) and (3) are close to $0^{\circ}$, and approximate the secant function by a second-order Taylor expansion.

Since $\mathbf{r}_{i p}$ and $\mathbf{r}_{t p}$ are now assumed to be nearly parallel to $\widehat{\mathbf{k}}_{0}$, we see that they are approximately scaled versions of $\mathbf{r}_{0}$, the original line of sight path vector. We therefore drop the boldface vector notation and use the scalar equivalent. We introduce scalars $\alpha_{p}, \beta_{p} \in[0,1]$ such that $r_{i p} \approx \alpha_{p} r_{0}$ and $r_{t p} \approx \beta_{p} r_{0}$. With these small angle approximations, we can write the condition for the memory effect $\widetilde{\Delta L}_{p}=\Delta L_{p}$ using equations (2) and (3) as

$$
\alpha_{p} \Delta \theta_{i p}^{2}+\beta_{p} \Delta \theta_{t p}^{2}=\Delta \theta_{i}^{2} .
$$


Because there is only one direct wave in a path summation, $\Delta \theta_{i}$ is an unknown constant, whereas $\Delta \theta_{i p}$ and $\Delta \theta_{t p}$ vary with scatterer position along the line of sight. Assuming we know the location of our point source and all the scatterers, we can, in principle, determine $\Delta \theta_{i p}$. Thus, we have one equation and two unknowns: $\Delta \theta_{i}$ and $\Delta \theta_{t p}$. We would like to solve for the change in angle of transmission, $\Delta \theta_{t p}$, and we may do so using the following constraint. Under our current approximations and assumptions, the first and last scattering events lie along the line of sight. Is there a single point along this line such that the change in angle of incidence $\Delta \theta_{i p}$ is nearly constant, i.e., independent of the path $p$ ? We choose the unknown rotation point $r_{x}$ to be this point. Then, given $\left\langle\widehat{\mathbf{k}}_{0}, \delta \mathbf{r}\right\rangle=0$, we have $\Delta \theta_{i} \sim \delta r / r_{x}$ (see Fig. 1) and $\Delta \theta_{i p} \sim \delta r /\left(\alpha_{p} r_{0}\right)$. We seek the point $r_{x}$ along the line of sight such that the change in angle of incidence of the direct wave $\Delta \theta_{i}$ is as close as possible to the change in angle of incidence of the scattered wave $\Delta \theta_{i p}$ for the most paths $p$. This occurs when $r_{x}$ minimizes

$$
S\left(r_{x}\right)=\frac{1}{2} \sum_{p}\left(\alpha_{p} r_{0}-r_{x}\right)^{2} .
$$

Thus, the point $r_{x}$ about which the direct wave rotates is simply the average scatterer position along the line connecting the point source to the speckle spot; that is, $r_{x}=$ $\alpha_{a v} r_{0}$, where $\alpha_{a v}$ is the average value of $\alpha_{p}$ along the line of sight. For paths $p$ such that $r_{i p} \approx r_{x}$, we have $\Delta \theta_{i p} \approx \Delta \theta_{i}$, and from equation (4) we find

$$
\Delta \theta_{t p}=\sqrt{\frac{1-\alpha_{a v}}{\beta_{p}}} \Delta \theta_{i} .
$$

Let $D$ be the thickness of the scattering region along the line of sight and $\varepsilon:=D / L_{0}$. Then, for all paths $p$ such that $1-\left(\alpha_{p}+\beta_{p}\right) \leq \varepsilon$, we have $\Delta \theta_{t p}-\Delta \theta_{i}=O(\varepsilon)$, which goes to 0 as $\varepsilon$ goes to 0 . In other words, the phenomenon in which changes in the angles of incidence and transmission are equivalent occurs only for a very small subset of trajectories through the random medium where the first and last scattering events occur near the mean scatterer position along the line of sight. This result is the wellknown memory effect. The dependence upon the thickness of the random medium is explicit via the parameter $\varepsilon$. In particular, the memory effect is most pronounced for transmission through thin media, which is consistent with performed experiments (see, for example, $[4,14]$ ). Also note that our result does not impose any restrictions on the random scattering trajectories occurring between the first and last scattering events. Thus, in this context, much of the scattering that goes on inside the random medium is completely arbitrary (see Fig. 2).

We next consider the temporal effects of a pulse source (i.e., a source of finite duration). In this case, the transmitted speckle pattern is a time varying function of path length. While we have shown the memory effect to hold in general for any trajectory that satisfies $\alpha+\beta \approx 1$, only for paths $p$ that also satisfy the subsidiary condition $\Delta L_{p}<\lambda / 4$, where $\lambda$ is the wavelength, will the scattered waves interfere coherently in time. That is, forward-scattered waves that pass through the first Fresnel zone will interefere with the direct wave, whereas multiple-scattered waves that perform a random walk will not interfere with the direct wave at all. For a pulse source, therefore, only the ballistic waves contribute to the shift in the speckle pattern. On the other hand, if the source is of an infinite duration (i.e., a continuous-wave source), at any given moment the speckle pattern is the simultaneous superposition of waves traveling along both ballistic and random walk trajectories, and both sets of trajectories contribute to the shift in the speckle pattern.

If one assumes the density of scatterers to be uniform, the maximum change in angle of incidence (and consequently the maximum change in angle of transmission) 




Fig. 2. A schematic illustrating two possible tracjectories through a random medium, shown as the shaded region, that contribute to the memory effect. The interference of the direct and scattered waves produce a speckle spot, shown as the dashed ellipse. The simplest forwardscattered trajectory is shown as the green dashed arrow. A random walk trajectory is shown as the purple dashed arrow. Both trajectories satisfy $\alpha+\beta \approx 1$.

for which the path vectors $\mathbf{r}_{i p}$ and $\mathbf{r}_{t p}$ remain within the same Fresnel zone is

$$
\Delta \theta_{\max } \sim \frac{W_{F}}{D},
$$

where $W_{F}$ is the width of the Fresnel zone determined by

$$
W_{F}=\sqrt{\frac{\lambda L_{0} \alpha \beta}{2}} .
$$

As the change in angle of incidence increases past $\Delta \theta_{\max }$, the path vectors $\mathbf{r}_{i p}$ and $\mathbf{r}_{t p}$ begin to pass through a different Fresnel zone - where the waves interact with different scatterers - and the resulting speckle pattern changes.

The extension of the memory effect to the case of an incident plane wave can be made by considering the limit in which the point source is placed infinitely far from the scattering medium. Then, by definition, all incidence angles are the same, and the memory effect will hold for any line of sight between the incident plane wave and the transmitted speckle pattern, provided the location of the last scattering event is near the average scatterer position along the line of sight.

\section{Numerical simulations}

To test the validity of our derivation, we model the transmitted wave fields in a twodimensional Cartesian geometry using the method described by Groenenboom and Snieder [19]. To simulate the scattering medium, we randomly embed 100 isotropic point scatterers in a $1000 \mathrm{~m} \times 800 \mathrm{~m}$ region within a constant velocity background $\left(c=2000 \mathrm{~ms}^{-1}\right)$. The scattering medium is placed $8000 \mathrm{~m}$ from a point source initially located at the origin (Fig. 3). A set $S=\left\{s_{0}, s_{1}, \ldots, s_{100}\right\}$ of receivers is placed 


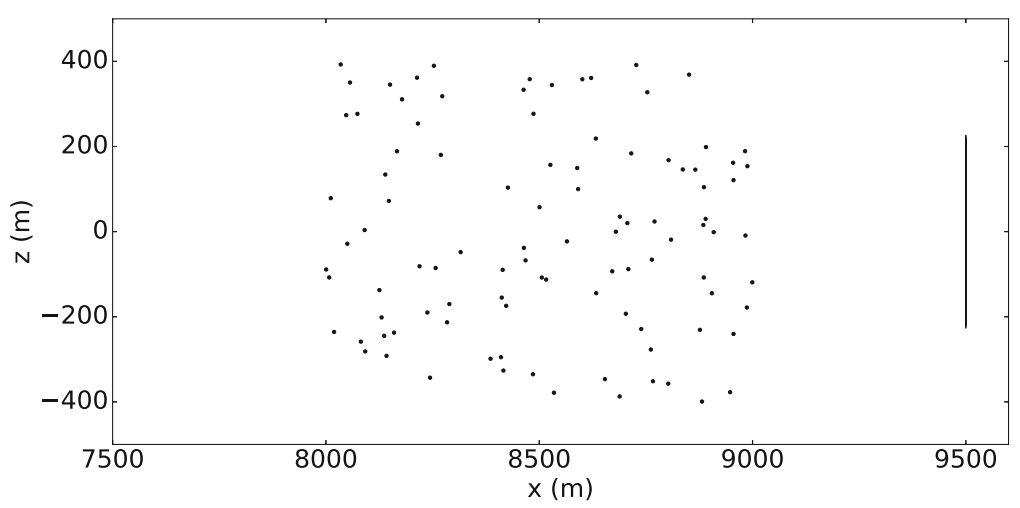

Fig. 3. A homogeneous velocity model with 100 isotropic point scatterers randomly embedded in a $1000 \mathrm{~m} \times 800 \mathrm{~m}$ region. The point source is located at $(x, z)=(0 \mathrm{~m}, 0 \mathrm{~m})$. A line of 101 receivers extends from $(9500 \mathrm{~m}, \pm 200 \mathrm{~m})$ at 4 -meter spacing.

behind the scattering medium between $(9500 \mathrm{~m}, \pm 200 \mathrm{~m})$ at 4 -meter spacing. The point source emits a single pulse with a bandwidth of $10 \mathrm{~Hz}$ to $80 \mathrm{~Hz}$ and central frequency $\nu_{0}=45 \mathrm{~Hz}$ (dominant period $T_{0}=2.2 \times 10^{-2} \mathrm{~s}$ ). We perturb the source in the vertical direction in $\pm 100 \mathrm{~m}$ increments up to a maximum displacement of $\delta r= \pm 1400 \mathrm{~m}$. For each value of $\delta r$, the source emits a pulse which transmits through the scattering medium, producing multiple-scattered waves that are recorded at each receiver for $367 T_{0}$.

With this model geometry, the mean scatterer position $\mathbf{r}_{x}$ along the line of sight between the origin and the midpoint of the receiver line is approximately located at $(8700 \mathrm{~m}, 0 \mathrm{~m})$. Using equation (8), the width of the Fresnel zone is $W_{F} \approx 128 \mathrm{~m}$, leading to a maximum change in angle of incidence $\Delta \theta_{\max } \approx 7.3^{\circ}$. We therefore expect the memory effect to break down when $|\delta r| / r_{x} \sim \Delta \theta_{\text {max }}$, or $|\delta r| \approx 1100 \mathrm{~m}$.

To measure the similarity of the transmitted wave fields produced for each $\delta r$, we compute time-shifted cross-correlations between the reference $(\delta r=0)$ and perturbed $(\delta r \neq 0)$ wave fields $\psi$ and $\widetilde{\psi}$ at each receiver position. Furthermore, we apply three different time windows to measure the range of the memory effect in various parts of the (time-domain) wave field: the full waveform (i.e., the entire wave field recording), the ballistic wave (window length $=11 T_{0}$ ), and the coda (window length $=130 T_{0}$ ). These time windows are illustrated in Figure 4. The correlation coefficient $C_{i j}^{\delta r}(\tau)$ is given by

$$
C_{i j}^{\delta r}(\tau)=\frac{\int \psi_{i}(t) \widetilde{\psi}_{j}(t+\tau) d t}{\sqrt{\int \psi_{i}^{2}(t) d t \int \widetilde{\psi}_{j}^{2}(t) d t}}, \quad-1 \leq C_{i j}^{\delta r}(\tau) \leq 1
$$

where the integration is over the time window under consideration. For each receiver pair $(i, j)$, we plot the correlation coefficient at the time shift $\tau^{*}$ for which $C_{i j}^{\delta r}\left(\tau^{*}\right)$ attains its maximum value. In the special case $\delta r=0 \mathrm{~m}, C_{i j}^{0}\left(\tau^{*}\right)=1$ when $i=j$ (i.e., the auto-correlation). We define the mean correlation coefficient $\langle C\rangle$ and mean speckle shift $\langle\delta s\rangle$ for each value of $\delta r$ as

$$
\begin{gathered}
\langle C\rangle=: \frac{1}{|S|} \sum_{i \in S} \max \left(\left\{C_{i j}^{\delta r}\left(\tau^{*}\right): j \in S\right\}\right), \\
\langle\delta s\rangle=: \frac{\Delta z}{|S|} \sum_{i \in S}\left(\underset{j}{\arg \max } C_{i j}^{\delta r}\left(\tau^{*}\right)-\underset{j}{\arg \max } C_{i j}^{0}\left(\tau^{*}\right)\right),
\end{gathered}
$$

where $|S|$ is the number of receivers and $\Delta z$ is the receiver spacing. 


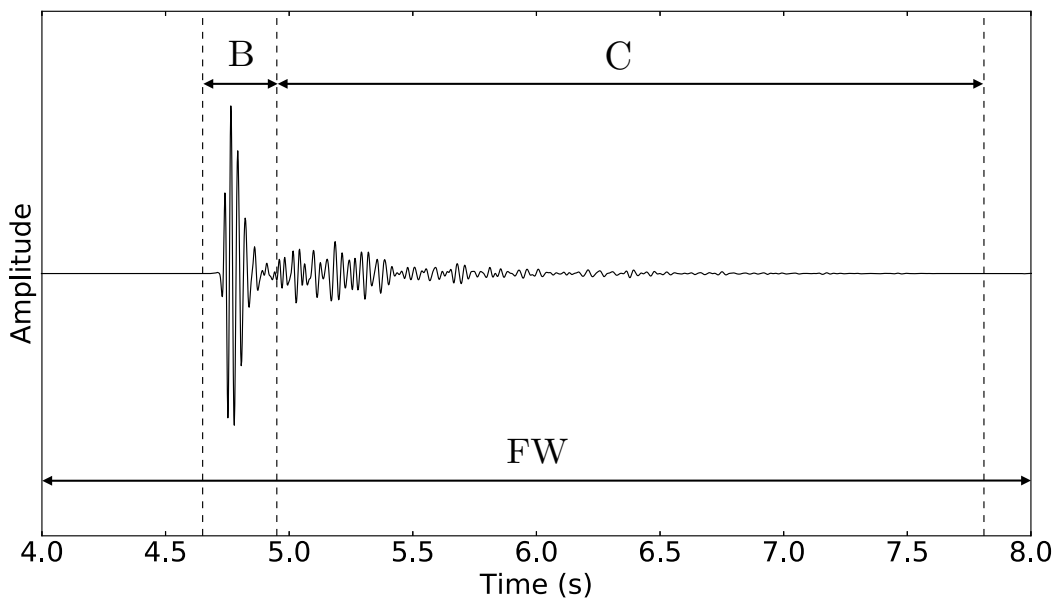

Fig. 4. A typical wave field recorded at one of the receivers. Forward-scattered waves that arrive early in the waveform constitute the ballistic arrival (time window B). Multiplescattered waves that arrive later in the waveform constitute the coda (time window $\mathrm{C}$ ). The full waveform is indicated by FW.

\section{Discussion of the numerical simulations}

Examples of the correlation (9) are shown in Figure 5 for the full waveform, ballistic, and coda time windows, respectively, for $\delta r=0 \mathrm{~m}$ and $\delta r=500 \mathrm{~m}$. Within each plot, the rows represent receiver $i$ measuring the reference wave field $\psi$, the columns represent receiver $j$ measuring the perturbed wave field $\widetilde{\psi}$, and the color values represent $C_{i j}^{\delta r}\left(\tau^{*}\right)$. The patterns in both the full waveform and ballistic correlation plots are nearly identical, and simply show that the pattern shifts (or translates) across receivers as $\delta r$ changes. The pattern in the coda correlation, however, is different from those in the full waveform and ballistic correlations. Furthermore, there is no coherent shift in the coda pattern as $\delta r$ is changed; rather, we see the values of the correlation function randomly fluctuate as $\delta r$ changes. [To see animations of these correlation experiments, see website.]

The mean correlation coefficient and mean speckle shift as functions of $\delta r$ (Eq. 10) are shown in Figure 6. The full waveform, ballistic, and coda time-windowed correlations all show that the mean correlation coefficient $\langle C\rangle$ decays to an average background value as $\delta r$ is increased. Note that the decay of $\langle C\rangle$ for the full waveform and the ballistic correlations is not symmetric about $\delta r=0 \mathrm{~m}$, but the decay of $\langle C\rangle$ for the coda correlation is almost perfectly symmetric.

A simple explanation for this observation is the nonuniform scatterer density of our model (Fig. 3); that is, the perturbations in the wave field when $\delta r>0$ are different than those when $\delta r<0$. Thus, the scatterer density appears to affect the rate of decay of the memory effect with increasing $\delta r$ (increasing $\Delta \theta$ ), suggesting the relation ( 7 ) does not hold when there are significant variations in scatterer density. In a medium with a uniform density of scatterers, however, we expect the decay of the memory effect to be approximately symmetric about $\delta r=0 \mathrm{~m}\left(\Delta \theta=0^{\circ}\right)$. The symmetry in the falloff of the coda correlation can be attributed to the fact that nonballistic waves traverse much longer paths throughout the scattering medium, thereby averaging out variations in the density of scatterers.

The right-hand column of Figure 6 shows the mean speckle shift $\langle\delta s\rangle$ versus $\delta r$ for the full waveform, ballistic, and coda correlations. The negative slope about $\delta r=0 \mathrm{~m}$ for the full waveform and ballistic correlations confirms that the transmitted speckle 


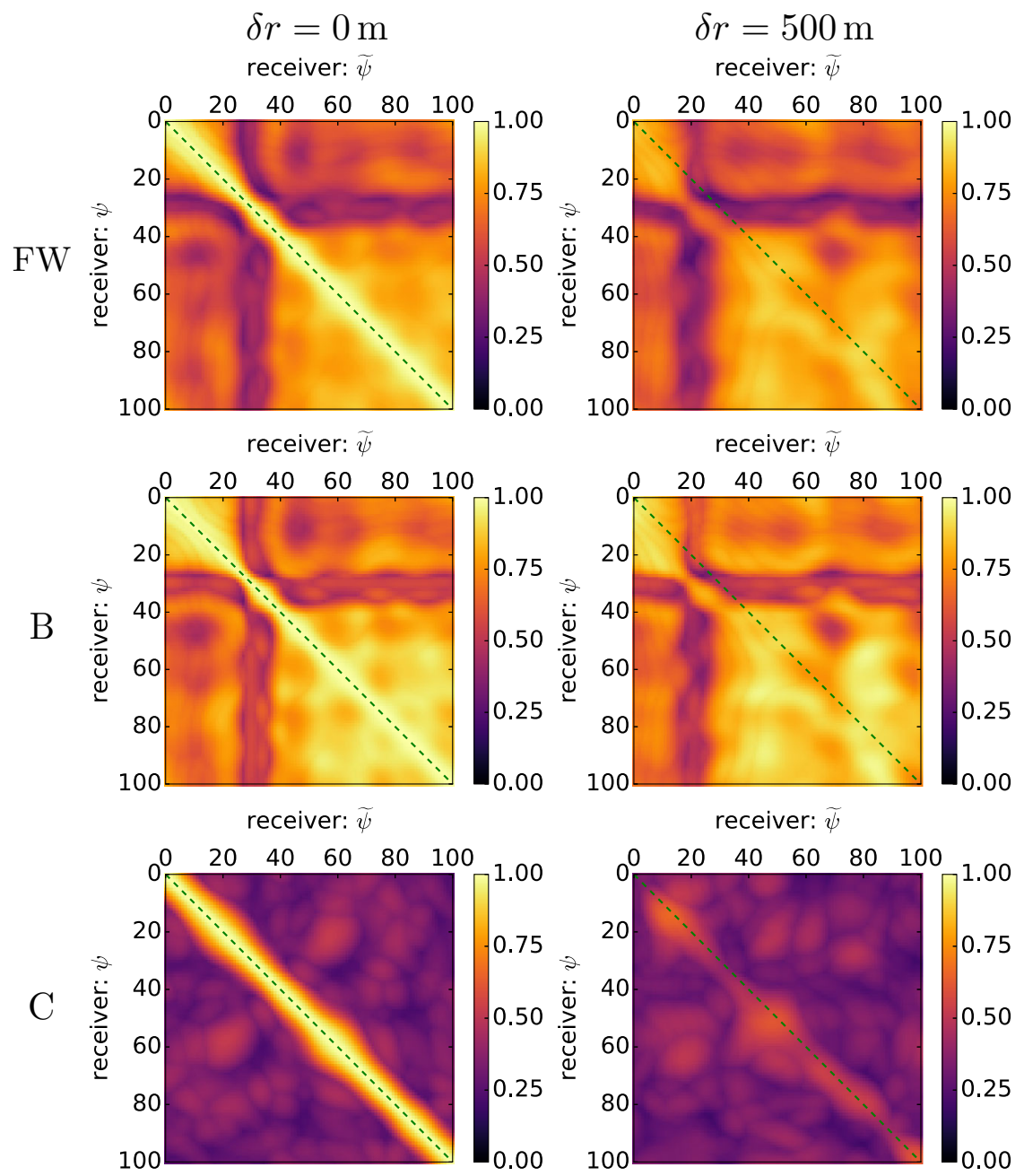

Fig. 5. Cross-correlation plots showing $C_{i j}^{\delta r}\left(\tau^{*}\right)$ for the full waveform FW, ballistic B, and coda $\mathrm{C}$ time windows. Plots are shown for $\delta r=0 \mathrm{~m}$ (left column) and $\delta r=500 \mathrm{~m}$ (right column).

pattern shifts in the direction opposite to the source perturbation. The value of $\delta r$ at which the slope changes from negative to positive indicates the maximum range of the memory effect. Note how the slope becomes positive near $\delta r=1100 \mathrm{~m}$ in the plot for the full waveform correlation, in agreement with our prediction. The near-horizontal slope in the coda correlation about $\delta r=0 \mathrm{~m}$ confirms that there is no coherent speckle shift for the coda waves.

\section{Conclusions}

We have shown that the memory effect can be derived by requiring phase differences to be conserved after a perturbation in the incident wave. From this conservation argument, we found the memory effect to hold for any trajectory through a random medium where the first and last scattering events occur near the mean scatterer position along the line of sight, as illustrated in Figure 2. Thus, the memory effect is 
$\langle C\rangle$
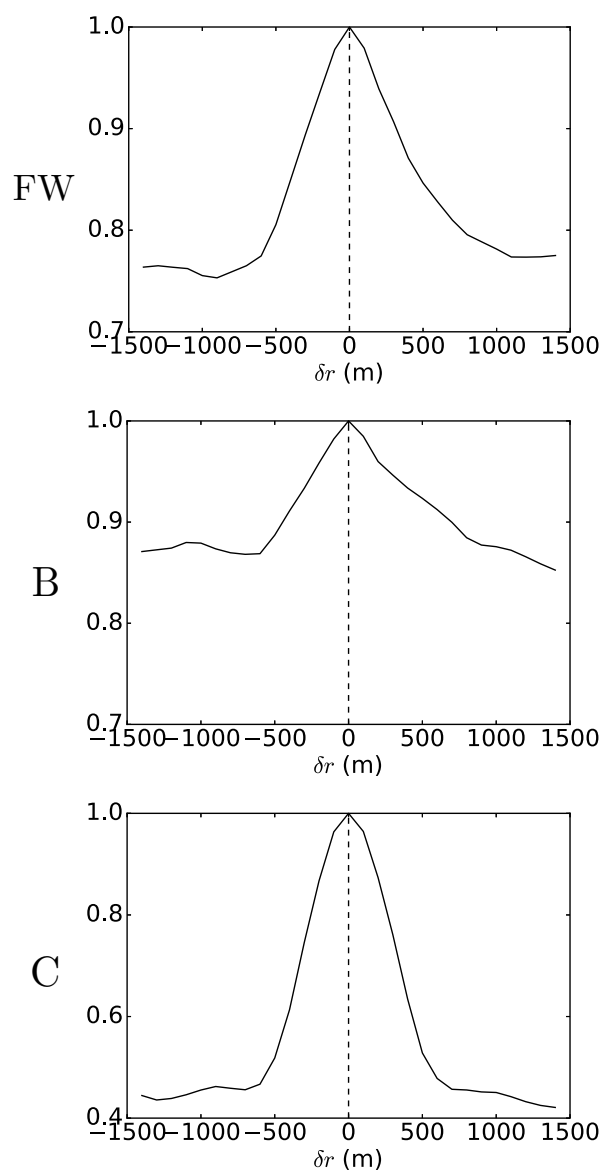

$\langle\delta s\rangle$
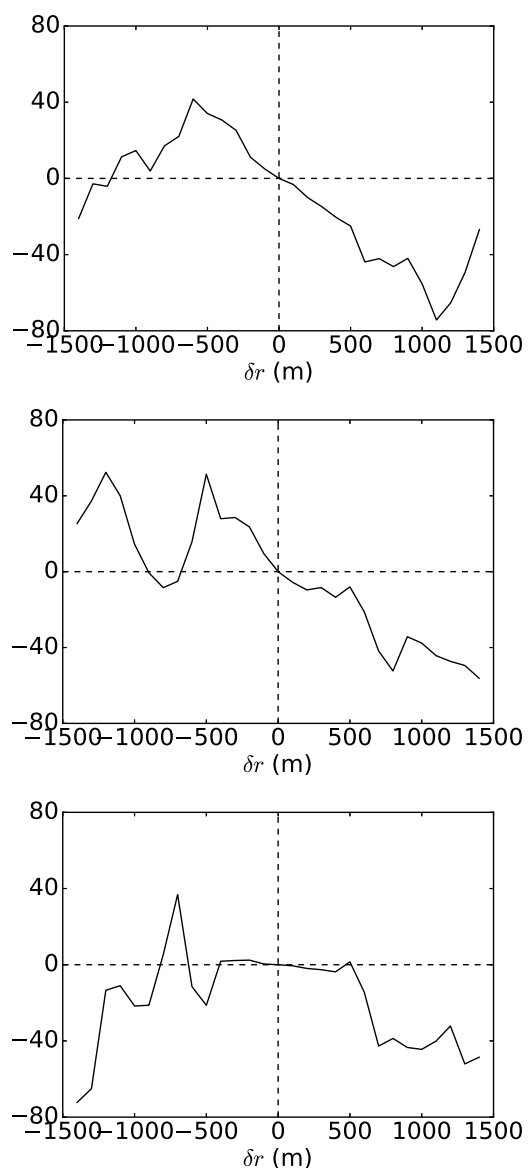

Fig. 6. Mean correlation coefficient $\langle C\rangle$ (left column) and mean speckle shift $\langle\delta s\rangle$ (right column) as functions of $\delta r$ for the full waveform $\mathrm{FW}$, ballistic $\mathrm{B}$, and coda $\mathrm{C}$ time-winodwed correlations.

independent of the phases acquired through the multiple scattering process, in agreement with established theory [2]. Our numerical simulations of pulse transmission through a random medium illustrate the temporal effects of the geometrical constraints imposed on the scattering trajectories that contribute to the memory effect. The results of our numerical simulations suggest that imaging through thin, random media with a pulse source can be achieved by exploiting the ballistic component of the transmitted wave field.

We would like to thank two anonymous reviewers, whose comments and critique greatly helped us to enhance the presentation and clarity of this paper. This work was supported by the Consortium Project on Seismic Inverse Methods for Complex Structures at the Colorado School of Mines.

\section{References}

1. S. Feng, C. Kane, P.A. Lee, A.D. Stone, Phys. Rev. Lett. 61, 834 (1988)

2. R. Berkovits, M. Kaveh, S. Feng, Phys. Rev. B. 40, 737 (1989) 
3. J.W. Goodman, Statistical Properties of Laser Speckle Patterns (Springer, Berlin, Heidelberg, 1975) pp. 9-75

4. I. Freund, M. Rosenbluh, S. Feng, Phys. Rev. Lett. 61, 2328 (1988)

5. I. Freund, M. Rosenbluh, R. Berkovits, Phys. Rev. B. 39, 12403 (1989)

6. R. Berkovits, M. Kaveh, Phys. Rev. B. 41, 7308 (1990)

7. R. Berkovits, M. Kaveh, J. Phys.: Condens. Matter. 2, 307 (1990)

8. R. Berkovits, M. Kaveh, Phys. Rev. B. 41, 2635 (1990)

9. R. Berkovits, M. Kaveh, Europhys. Lett. 13, 97 (1990)

10. I. Freund, R. Berkovits, Phys. Rev. B. 41, 496 (1990)

11. R. Berkovits, S. Feng, Phys. Rep. 238, 135 (1994)

12. A. Kurita, Y. Kanematsu, M. Watanabe, K. Hirata, T. Kushida, Phys. Rev. Lett. 83, $1582(1999)$

13. I.M. Vellekoop, C.M. Aegerter, Opt. Lett. 35, 1245 (2010)

14. J. Bertolotti, E.G. van Putten, C. Blum, A. Lagendijk, W.L. Vos, A.P. Mosk, Nature 491, $232(2012)$

15. O. Katz, E. Small, Y. Silberberg, Nat. Photonics 6, 549 (2012)

16. O. Katz, P. Heidmann, M. Fink, S. Gigan, Nat. Photonics 8, 784 (2014)

17. B. Judkewitz, R. Horstmeyer, I.M. Vellekoop, I.N. Papadopoulos, C. Yang, Nat. Phys. 11, $684(2015)$

18. J. Bertolotti, Nat. Phys. 11, 622 (2015)

19. J. Groenenboom, R. Snieder, J. Acoust. Soc. Am. 98, 3482 (1995)

20. N. Bleistein, Mathematical Methods for Wave Phenomena (Academic Press, Orlando, 1984) pp. $77-81$

Open Access This is an Open Access article distributed under the terms of the Creative Commons Attribution License (http://creativecommons.org/licenses/by/4.0), which permits unrestricted use, distribution, and reproduction in any medium, provided the original work is properly cited. 